\title{
Correction to: Academic Pain Medicine
}

\author{
Yury Khelemsky, Anuj Malhotra, and Karina Gritsenko
}

\section{Correction to: \\ Y. Khelemsky et al. (eds.), Academic Pain Medicine, https://doi.org/10.1007/978-3-030-18005-8}

The book was inadvertently published with incorrect authorships in Chapters 13, 14 and 47 . It has been updated along with corresponding affiliation information as follows:

Chapter 13:

From "Karina Gritsenko" to "Ricardo Maturana, Andrew So, and Karina Gritsenko"

Ricardo Maturana

Albert Einstein College of Medicine, Montefiore Medical Center, Bronx, NY, USA

Andrew So

Albert Einstein College of Medicine, Montefiore Medical Center, Bronx, NY, USA

Karina Gritsenko

Department of Anesthesiology, Montefiore Medical Center and Albert Einstein College of Medicine, Bronx, NY, USA

Chapter 14:

From "Melinda Aquino" to "Chukwuemeka Okafor and Melinda Aquino"

Chukwuemeka Okafor

Forbes Hospital, Pittsburgh, Pennsylvania, USA

Melinda Aquino

Montefiore Medical Center, Albert Einstein College of Medicine, Bronx, NY, USA

Chapter 47:

From "Boleslav Kosharskyy" to "Guensley R. Delva, Jacquelyn K. Francis, and Demetri Koutsospyros"

Guensley R. Delva

Dartmouth Hitchcock Medical Center, Theodore Geisel School of Medicine, Lebanon, NH, USA

Jacquelyn K. Francis

Hackensack University Medical Center, Hackensack, NJ, USA

Demetri Koutsospyros

Department of Anesthesiology, Montefiore Medical Center, Bronx, NY, USA

The updated versions of the chapters can be found at https://doi.org/10.1007/978-3-030-18005-8_13 https://doi.org/10.1007/978-3-030-18005-8_14 https://doi.org/10.1007/978-3-030-18005-8_47 